\title{
Hamiltonian Approach to the Dynamical Casimir Effect
}

\author{
Jaume Haro $^{1, *}$ and Emilio Elizalde ${ }^{2, \dagger}$ \\ ${ }^{1}$ Departament de Matemàtica Aplicada I, Universitat Politècnica de Catalunya, Diagonal 647, 08028 Barcelona, Spain \\ ${ }^{2}$ Instituto de Ciencias del Espacio (CSIC) and Institut d'Estudis Espacials de Catalunya (IEEC/CSIC), Facultat de Ciències, \\ Universitat Autònoma de Barcelona, Torre C5-Parell-2a Planta, 08193 Bellaterra (Barcelona), Spain
}

(Received 24 July 2006; published 25 September 2006)

\begin{abstract}
A Hamiltonian approach is introduced in order to address some severe problems associated with the physical description of the dynamical Casimir effect at all times. For simplicity, the case of a neutral scalar field in a one-dimensional cavity with partially transmitting mirrors (an essential proviso) is considered, but the method can be extended to fields of any kind and higher dimensions. The motional force calculated in our approach contains a reactive term - proportional to the mirrors' acceleration - which is fundamental in order to obtain (quasi)particles with a positive energy all the time during the movement of the mirrors - while always satisfying the energy conservation law. Comparisons with other approaches and a careful analysis of the interrelations among the different results previously obtained in the literature are carried out.
\end{abstract}

Introduction. - Moving mirrors modify the structure of the quantum vacuum, what manifests in the creation and annihilation of particles. Once the mirrors return to rest, a number of the produced particles may still remain, which can be interpreted as radiated particles. This flux has been calculated in several situations by using different methods, as averaging over fast oscillations [1,2], by multiple scale analysis [3], with the rotating wave approximation [4], with numerical techniques [5], and others [6]. Here we will be interested in the production of the particles and their possible energy all the time while the mirrors are moving. In the case of a single, perfectly reflecting mirror, the number of produced particles as well as their energy diverge while the mirror moves. Several renormalization prescriptions have been used in order to obtain a welldefined energy; however, for some trajectories this finite energy is not a positive quantity and cannot be identified with the energy of the produced particles (see, e.g., [7]).

Our approach relies on two basic ingredients: proper use of a Hamiltonian method and the consideration of partially transmitting mirrors, which become transparent to very high frequencies. We shall prove, in this way, both that the number of created particles is finite and also that their energy is always positive for the whole trajectory corresponding to the mirrors' displacement. We will also calculate the radiation-reaction force that acts on the mirrors owing to the emission and absorption of particles, which is related with the field's energy through the energy conservation law, so that the energy of the field at any time $t$ is equal, with opposite sign, to the work performed by the reaction force up to time $t[8,9]$. Such force is usually split into two parts [10,11]: a dissipative force whose work equals minus the energy of the particles that remain [8], and a reactive force vanishing when the mirrors return to rest. We will show that the radiation-reaction force calculated from the Hamiltonian approach for partially transmitting mirrors satisfies, at all time, the energy conservation law and can naturally account for the creation of positive energy particles. Also, the dissipative part we will obtain agrees with the one calculated by other methods, as using the Heisenberg picture or other effective Hamiltonians. Note that those methods have problems with the reactive part, which in general yields a nonpositive energy that cannot be considered as that of the particles created at any $t$.

In what follows, we first introduce the Hamiltonian method for a neutral Klein-Gordon field in a cavity with boundaries moving at a certain speed $v \ll c$. Then, a single partially transmitting mirror in $1+1$ spacetime will be studied in order to illustrate the procedure and prove the above statements. Our results will be compared with the ones in the literature. Finally, the case of two mirrors will be investigated to see that also here we obtain physically meaningful quantities while the mirrors move, in an unambiguous way, and that the dissipative force does agree with previous results by other authors.

The Hamiltonian formulation. - Consider a neutral massless scalar field in a cavity $\Omega_{t}$, and assume that the boundary is at rest for time $t \leq 0$ and returns to its initial position at time $T$. Suppose also its velocity to be of order $\epsilon=v / c$ (dimensionless, it is of order $10^{-8}$ in [12]; see later). The Lagrangian density of the field is $\mathcal{L}(t, \mathbf{x})=\frac{1}{2} \times$ $\left[\left(\partial_{t} \phi\right)^{2}-\left|\nabla_{\mathbf{x}} \phi\right|^{2}\right], \mathbf{x} \in \Omega_{t} \subset \mathbb{R}^{3}, t \in \mathbb{R}$. In terms of the canonical conjugated momentum $\xi(t, \mathbf{x}) \equiv \frac{\partial \mathcal{L}}{\partial\left(\partial_{t} \phi\right)}=$ $\partial_{t} \phi(t, \mathbf{x})$, the energy density of the field is $\mathcal{E}(t, \mathbf{x}) \equiv$ $\xi \partial_{t} \phi-\mathcal{L}(t, \mathbf{x})=\frac{1}{2}\left(\xi^{2}+\left|\nabla_{\mathbf{x}} \phi\right|^{2}\right)$, while its energy is $E(t ; \epsilon) \equiv \int_{\Omega_{t}} d^{3} \mathbf{x} \mathcal{E}(t, \mathbf{x})$. As is well known, this energy density does not coincide with the Hamiltonian one [1315]. The Hamiltonian density can be conveniently obtained using the method in [16].

First, a (nonconformal) coordinate change is used to convert the moving boundary $\Omega_{t}$ into a fixed one $\tilde{\Omega}$ : 
$(t(\bar{t}, \mathbf{y}), \mathbf{x}(\bar{t}, \mathbf{y}))=\mathcal{R}(\bar{t}, \mathbf{y})=(\bar{t}, \mathbf{R}(\bar{t}, \mathbf{y}))(\bar{t}$ the new time $)$. The action of the system is $S=\int_{\mathbb{R}} \int_{\tilde{\Omega}} d^{3} \mathbf{y} d \bar{t} \tilde{\mathcal{L}}(\bar{t}, \mathbf{y})$, with $\tilde{\mathcal{L}}(\bar{t}, \mathbf{y}) \equiv J \mathcal{L}(\mathcal{R}(\bar{t}, \mathbf{y}))$, with $J$ being the Jacobian of the change, $d^{3} \mathbf{x} \equiv J d^{3} \mathbf{y}$. For the function $\tilde{\phi}(\bar{t}, \mathbf{y}) \equiv$ $\sqrt{J} \phi(\mathcal{R}(\bar{t}, \mathbf{y}))$, the conjugated momentum is $\tilde{\xi}(\bar{t}, \mathbf{y}) \equiv$ $\frac{\partial \tilde{\mathcal{L}}}{\partial\left(\partial_{\tilde{t}} \tilde{\phi}\right)}=\sqrt{J} \partial_{t} \phi(\mathcal{R}(\bar{t}, \mathbf{y}))$, and the Hamiltonian density

$$
\tilde{\mathcal{H}}(\bar{t}, \mathbf{y})=\frac{1}{2}\left(\tilde{\xi}^{2}+J\left|\boldsymbol{\nabla}_{\mathbf{x}} \phi\right|^{2}\right)+\tilde{\xi}\left(\partial_{\bar{t}} \tilde{\phi}-\sqrt{J} \partial_{t} \phi\right) .
$$

In the coordinates $(t, \quad \mathbf{x})$ after some calculations, $\mathcal{H}(t, \mathbf{x})=\mathcal{E}(t, \mathbf{x})+\xi(t, \mathbf{x})\left\langle\partial_{s} \mathbf{R}\left(\mathcal{R}^{-1}(t, \mathbf{x})\right), \nabla_{\mathbf{x}} \phi(t, \mathbf{x})\right\rangle+$ $\left.\frac{1}{2} \xi(t, \mathbf{x}) \phi(t, \mathbf{x}) \partial_{s}(\ln J)\right|_{\mathcal{R}^{-1}(t, \mathbf{x})}$. For a single mirror which follows a prescribed trajectory $[\epsilon g(t), t]$ in $1+1$ spacetime, we can set $R(\bar{t}, y)=y+\epsilon g(\bar{t})$, and obtain $\mathcal{H}(t, x)=$ $\mathcal{E}(t, x)+\epsilon \dot{g}(t) \xi(t, x) \partial_{x} \phi(t, x)$.

Case of a single, partially transmitting mirror.-We here consider a single mirror in $1+1$ spacetime, following a prescribed trajectory $[t, \epsilon g(t)]$. When the mirror is at rest, scattering is described by the matrix

$$
S(\omega)=\left(\begin{array}{cc}
s(\omega) & r(\omega) e^{-2 i \omega L} \\
r(\omega) e^{2 i \omega L} & s(\omega)
\end{array}\right),
$$

where $x=L$ is the position of the mirror. The $S$ matrix is taken to be real in the temporal domain, causal, unitary, and the identity at high frequencies [17]. Specifically, (i) $S(-\omega)=S^{*}(\omega)$, (ii) $S(\omega)$ is analytic for $\operatorname{Im}(\omega)>0$, with $s(\omega)$ and $r(\omega)$ being meromorphic (cutoff) functions (the material's permitivity and resistivity), (iii) $S(\omega) S^{\dagger}(\omega)=$ Id, and (iv) $S(\omega) \rightarrow$ Id, when $|\omega| \rightarrow \infty$.

To reach the quantum theory from the Hamiltonian approach, we set the mirror at $y=0$ in the above coordinates; the right and left incident modes are $\tilde{g}_{\omega, R}(y)=$ $\frac{1}{\sqrt{4 \pi \omega}}\left\{s(\omega) e^{-i \omega y} \theta(-y)+\left[e^{-i \omega y}+r(\omega) e^{i \omega y}\right] \theta(y)\right\} \quad$ and $\tilde{g}_{\omega, L}(y)=\frac{1}{\sqrt{4 \pi \omega}}\left\{\left[e^{i \omega y}+r(\omega) e^{-i \omega y}\right] \theta(-y)+s(\omega) e^{i \omega y} \theta(y)\right\}$. In the coordinates $(t, x)$ the instantaneous set of the right and left incident eigenfunctions which generalize the set for a perfectly reflecting mirror is $g_{\omega, j}(t, x ; \epsilon) \equiv \tilde{g}_{\omega, j}(x-$ $\epsilon g(t)), j=R, L$. In general, we do not know which is the part of the Hamiltonian that describes the interaction between the field and the mirror. To get the quantized theory, the energy of the field $E(t)=\int_{\mathbb{R}} d x \mathcal{E}(t, x)$, which in the presence of a single mirror does not depend on $\epsilon$, must be considered as part of the free Hamiltonian of the system. In the interaction picture, the Schrödinger equation is $i \partial_{t}|\Phi\rangle=\epsilon \dot{g}(t) \int_{\mathbb{R}} d x \hat{\xi}_{I}(t, x ; \epsilon) \partial_{x} \hat{\phi}_{I}(t, x ; \epsilon)|\Phi\rangle=\epsilon \dot{g}(t) \times$ $\int_{\mathbb{R}} d x \hat{\xi}_{I}(t, x ; 0) \partial_{x} \hat{\phi}_{I}(t, x ; 0)|\Phi\rangle+\mathcal{O}\left(\epsilon^{2}\right)$. The average number of (quasi)particles [18] and the dynamical energy (e.g., the energy of the created particles) at time $t$ are, respectively,

$$
\begin{array}{r}
\mathcal{N}(t) \equiv \sum_{j=R, L} \int_{0}^{\infty} d \omega\left\langle 0\left|\left(\mathcal{T}^{t}\right)^{\dagger} \hat{a}_{\omega, j}^{\dagger} \hat{a}_{\omega, j} \mathcal{T}^{t}\right| 0\right\rangle, \\
\langle\hat{E}(t)\rangle \equiv \sum_{j=R, L} \int_{0}^{\infty} d \omega \omega\left\langle 0\left|\left(\mathcal{T}^{t}\right)^{\dagger} \hat{a}_{\omega, j}^{\dagger} \hat{a}_{\omega, j} \mathcal{T}^{t}\right| 0\right\rangle,
\end{array}
$$

with $\mathcal{T}^{t}$ being the quantum evolution operator. A simple but cumbersome calculation yields the following results:

$$
\begin{aligned}
\mathcal{N}(t)= & \frac{\epsilon^{2}}{2 \pi^{2}} \int_{0}^{\infty} \int_{0}^{\infty} \frac{d \omega d \omega^{\prime} \omega \omega^{\prime}}{\left(\omega+\omega^{\prime}\right)^{2}}\left|\widehat{\dot{g}}_{t}\left(\omega+\omega^{\prime}\right)\right|^{2}[\mid r(\omega) \\
+ & \left.\left.r^{*}\left(\omega^{\prime}\right)\right|^{2}+\left|s(\omega)-s^{*}\left(\omega^{\prime}\right)\right|^{2}\right]+\mathcal{O}\left(\epsilon^{4}\right), \\
\langle\hat{E}(t)\rangle= & \frac{\epsilon^{2}}{4 \pi^{2}} \int_{0}^{\infty} \int_{0}^{\infty} \frac{d \omega d \omega^{\prime} \omega \omega^{\prime}}{\left(\omega+\omega^{\prime}\right)}\left|\widehat{g}_{t}\left(\omega+\omega^{\prime}\right)\right|^{2}[\mid r(\omega) \\
& \left.+\left.r^{*}\left(\omega^{\prime}\right)\right|^{2}+\left|s(\omega)-s^{*}\left(\omega^{\prime}\right)\right|^{2}\right]+\mathcal{O}\left(\epsilon^{4}\right),
\end{aligned}
$$

where $\theta_{t}$ is the Heavyside step function, $\theta_{t}(\tau)=\theta(t-\tau)$, and $\hat{f}$ the Fourier transform of $f$.

These two quantities are in general convergent. However, for the seminal Davis-Fulling model [7] of a single, perfectly reflecting mirror, both quantities diverge when the mirror moves or when its movement has discontinuities of some kind $[15,19]$. To obtain a finite energy, different regularization techniques have been used. For instance, with a frequency cutoff $e^{-\gamma \omega}$, with $0<\gamma \ll 1$, the regularized energy is $\langle\hat{E}(t ; \gamma)\rangle=\frac{\epsilon^{2}}{6 \pi}\left[\frac{\dot{g}^{2}(t)}{\pi \gamma}-\ddot{g}(t) \dot{g}(t)+\right.$ $\left.\int_{0}^{t} \ddot{g}^{2}(\tau) d \tau\right]$, and imposing that the kinetic energy of the moving boundary be $\frac{1}{2}\left(M_{\exp }-\frac{1}{3 \pi^{2} \gamma}\right) \epsilon^{2} \dot{g}^{2}(t)$, with $M_{\exp }$ the experimental mass of the mirror, some authors conclude that the renormalized dynamical energy, namely, $\hat{E}_{R}(t)$, is [7-10]

$$
\left\langle\hat{E}_{R}(t)\right\rangle \equiv \frac{\epsilon^{2}}{6 \pi}\left[-\ddot{g}(t) \dot{g}(t)+\int_{0}^{t} \ddot{g}^{2}(\tau) d \tau\right] .
$$

However, when $t \leq \delta$, with $0<\delta \ll 1$, this renormalized energy is negative, which shows that, while the mirror moves, the renormalized energy cannot be considered as the energy of the produced particles at time $t$ [cf. the paragraph after Eq. (4.5) in [7]]. We interpreted such results as implying that a perfectly reflecting mirror is nonphysical and decided to approach the problem by considering instead a partially transmitting mirror, transparent to high frequencies. Results are rewarding: in our Hamiltonian approach Eqs. (4) and (6), for the radiation-reaction force, e.g., the difference between the energy density of the evolved vacuum state on the left and right sides of the mirror, we do get the right sign

$$
\left\langle\hat{F}_{\mathrm{Ha}}(t)\right\rangle=-\frac{\epsilon}{2 \pi^{2}} \int_{0}^{\infty} \int_{0}^{\infty} \frac{d \omega d \omega^{\prime} \omega \omega^{\prime}}{\omega+\omega^{\prime}} \operatorname{Re}\left[e^{-i\left(\omega+\omega^{\prime}\right) t} \widehat{\dot{g} \theta_{t}}\left(\omega+\omega^{\prime}\right)\right]\left[\left|r(\omega)+r^{*}\left(\omega^{\prime}\right)\right|^{2}+\left|s(\omega)-s^{*}\left(\omega^{\prime}\right)\right|^{2}\right]+\mathcal{O}\left(\epsilon^{2}\right) .
$$

Note this integral diverges for a perfect mirror $(r \equiv-1, s \equiv 0$, ideal case), but nicely converges for our partially 
transmitting (physical) one where $r(\omega) \rightarrow 0, s(\omega) \rightarrow 1$, as $\omega \rightarrow \infty$ [see (2)]. Energy conservation is fulfilled: the dynamical energy at any time $t$ equals, with the opposite sign, the work performed by the reaction force up to that time $t[8,9]$; in fact, from (6) and (8),

$$
\langle\hat{E}(t)\rangle=-\epsilon \int_{0}^{t}\left\langle\hat{F}_{\mathrm{Ha}}(\tau)\right\rangle \dot{g}(\tau) d \tau
$$

Comparison with other results. - First, we have repeated the calculations using the Heisenberg picture approach of [20]. We have obtained the "in" modes when the mirror describes the prescribed trajectory $[t, \epsilon g(t)]$. Then, we have obtained the average number of produced particles after the mirror returns to rest [21]:

$$
\mathcal{N}(t \geq T)=\sum_{i, j=R, L} \int_{0}^{\infty} \int_{0}^{\infty} d \omega d \omega^{\prime}\left|\left(\phi_{\omega, i}^{\mathrm{out}}, \phi_{\omega^{\prime}, j}^{\mathrm{in} *}\right)\right|^{2},
$$

by calculating the Bogoliubov coefficients $\left(\phi_{\omega, i}^{\text {out }}, \phi_{\omega^{\prime}, j}^{\text {in* }}\right)$ in the null future infinity $I^{+}$(outgoing modes acquire a very simple expression in $I^{+}$). The final result turns out to be exactly the same expression (5).

The radiation-reaction force in the Heisenberg picture, $\left\langle\hat{F}_{H}(t)\right\rangle$, is the difference between the energy density of the in vacuum state on the left and right sides of the mirror. A simple calculation shows that the energy density on both sides of the mirror is $\langle\hat{\mathcal{E}}(t, x)\rangle=\int_{0}^{\infty} d \omega \omega \pm$ $\frac{i \epsilon}{8 \pi^{2}} \int_{\mathbb{R}^{2}} d \omega d \omega^{\prime} \omega \omega^{\prime} \hat{g}\left(\omega+\omega^{\prime}\right) \chi(\omega)\left[1+r(\omega) r\left(\omega^{\prime}\right)-\right.$ $\left.s(\omega) s\left(\omega^{\prime}\right)\right] e^{-i\left(\omega+\omega^{\prime}\right) v} \theta( \pm(\epsilon g(t)-x))+\mathcal{O}\left(\epsilon^{2}\right), \chi(\omega) \equiv$ $\theta(\omega)-\theta(-\omega)$ being the sign function. Note that the term of order $\epsilon$ is ill-defined, since the function $\omega \omega^{\prime} \hat{g}\left(\omega+\omega^{\prime}\right) \times$ $\left[1+r(\omega) r\left(\omega^{\prime}\right)-s(\omega) s\left(\omega^{\prime}\right)\right]$ is not Lebesgue integrable. Some regularization is needed to obtain a well-defined quantity. Defining the regularized energy by $\langle\hat{\mathcal{E}}(t, x ; \gamma)\rangle \equiv$ $\sum_{j=R, L} \int_{0}^{\infty} d \omega e^{-\gamma \omega}\left[\partial_{u} \phi_{\omega, j}^{\text {in }}(u, v ; \gamma) \partial_{u} \phi_{\omega, j}^{\text {in* }}(u, v ; \gamma)+\right.$ $\left.\partial_{v} \phi_{\omega, j}^{\text {in }}(u, v ; \gamma) \partial_{v} \phi_{\omega, j}^{\text {in* }}(u, v ; \gamma)\right]$, with the in modes regularized to obtain a cutoff independent quantity [22], in the Heisenberg picture reads $\left\langle\hat{F}_{H}(t ; \gamma)\right\rangle=\frac{i \epsilon}{8 \pi^{2}} \times$ $\int_{\mathbb{R}^{2}} d \omega d \omega^{\prime} \omega \omega^{\prime} \hat{g}\left(\omega+\omega^{\prime}\right)\left[\chi(\omega)+\chi\left(\omega^{\prime}\right)\right]\left[1+r(\omega) r\left(\omega^{\prime}\right)-\right.$ $\left.s(\omega) s\left(\omega^{\prime}\right)\right] e^{-\gamma\left(|\omega|+\left|\omega^{\prime}\right|\right)} e^{-i\left(\omega+\omega^{\prime}\right) t}+\mathcal{O}\left(\epsilon^{2}\right)$. This converges and is cutoff independent, and a possible definition of the renormalized radiation-reaction force is

$$
\begin{aligned}
\left\langle\hat{F}_{H, \text { ren }}(t)\right\rangle= & \frac{i \epsilon}{8 \pi^{2}} \int_{\mathbb{R}^{2}} d \omega d \omega^{\prime} \omega \omega^{\prime} \hat{g}\left(\omega+\omega^{\prime}\right) \\
& \times\left[\chi(\omega)+\chi\left(\omega^{\prime}\right)\right]\left[1+r(\omega) r\left(\omega^{\prime}\right)\right. \\
& \left.-s(\omega) s\left(\omega^{\prime}\right)\right] e^{-i\left(\omega+\omega^{\prime}\right) t}+\mathcal{O}\left(\epsilon^{2}\right) .
\end{aligned}
$$

In general, this formula disagrees with the radiationreaction force (8) which was obtained using the Hamiltonian approach. Moreover, we have been able to prove (details will be provided elsewhere [22]) that the force (11) coincides with the radiation-reaction force calculated by Jaekel and Reynaud [23] after renormalization: $\left\langle\hat{F}_{J, R \text {,ren }}(t)\right\rangle \equiv\left\langle\hat{F}_{H \text {,ren }}(t)\right\rangle$. We thus conclude that the method of Jaekel and Reynaud is equivalent to the quantum theory in the Heisenberg picture. Furthermore, note that $\epsilon \int_{\mathbb{R}} d t\left\langle\hat{F}_{\mathrm{Ha}}(t)\right\rangle \dot{g}(t)=\epsilon \int_{\mathbb{R}} d t\left\langle\hat{F}_{J, R, \text { ren }}(t)\right\rangle \dot{g}(t)$, and this identity proves that the dissipative parts of $\left\langle\hat{F}_{\mathrm{Ha}}(t)\right\rangle$ and $\left\langle\hat{F}_{J, R \text {,ren }}(t)\right\rangle$ always agree.

However, in several situations the reactive parts do not match. For instance, if $r(w)=-\frac{i \alpha}{\omega+i \alpha}$ and $s(w)=\frac{\omega}{\omega+i \alpha}$ with $\alpha>0$, there is the relation

$$
\left\langle\hat{F}_{\mathrm{Ha}}(t)\right\rangle=-\frac{\alpha \epsilon}{2 \pi} \ddot{g}(t)+\left\langle\hat{F}_{J, R, \text { ren }}(t)\right\rangle,
$$

where $\quad\left\langle\hat{F}_{J, R, \text { ren }}(t)\right\rangle=\frac{\alpha \epsilon}{\pi} \int_{1}^{\infty} d z \int_{-\infty}^{t} d \tau\left(z^{-2}-z^{-3}\right) \times$ $e^{-\alpha z(t-\tau)} \dddot{g}(\tau)$. The two forces differ in a reactive term. Now the crucial point is that, during the movement of the mirror, the work done by the motion force $\left\langle\hat{F}_{J, R, \text { ren }}(t)\right\rangle$ is not a negative quantity. Consequently, the dynamical energy is not positive and a meaningless result is obtained because the dynamical energy is the energy of the produced particle. To avoid this difficulty, the reactive term $-\frac{\alpha \epsilon}{2 \pi} \ddot{g}(t)$, which most naturally appears in the Hamiltonian formulation for a partially transmitting mirror, comes to rescue and renders a physically meaningful result.

Barton and Calogeracos [11] (see also [24]) studied the case $r(w)=-\frac{i \alpha}{\omega+i \alpha}, s(w)=\frac{\omega}{\omega+i \alpha}$, with $\alpha>0$. The interaction between field and mirror can be described there by the Lagrangian density $\frac{1}{2}\left[\left(\partial_{t} \phi\right)^{2}-\left(\partial_{x} \phi\right)^{2}\right]-\alpha \phi^{2} \delta(x-$ $\epsilon g(t))$. With the Hamiltonian method we have obtained the corresponding quantized Hamiltonian, and $\int_{\mathbb{R}} d x \hat{\mathcal{E}}(t, x)+$ $\alpha \hat{\phi}^{2}(t, \epsilon g(t))=\sum_{j=L, R} \int_{0}^{\infty} d \omega \omega\left(\hat{a}_{\omega, j}^{\dagger} \hat{a}_{\omega, j}+1 / 2\right)$. This leads, for these reflection and transmission coefficients, back to our formulas (5), (6), and (8). However, two important differences exist. First, to obtain the Schrödinger equation, these authors make a unitary transformation which does not seem easily generalizable to the case of two moving mirrors. And second, in [11], following $[9,10]$, a mass renormalization is performed - in order to eliminate the reactive part of the motion force-where the energy of the field is not a positive quantity at any time $t$. Again, the concept of particle is ill-defined during the mirror's displacement.

Two partially transmitting mirrors. -We have finally extended our method to the case of two moving mirrors that follow prescribed trajectories $\left[t, L_{j}(t ; \epsilon)\right]$, where $L_{j}(t ; \epsilon) \equiv L_{j}+\epsilon g_{j}(t)$, with $j=1,2$ assuming that $L_{1}(t ; \epsilon)<L_{2}(t ; \epsilon)$, for all $t \in \mathbb{R}$. In this case it is impossible, in practice, to work in the Heisenberg picture, because it is extremely difficult to obtain the in and out mode functions in the presence of the two moving mirrors. Instead, in order to get the dissipative part of the motion force, the number of radiated particles, and their energy, the approach of Jaekel and Reynaud can be used, which starts from the effective Hamiltonian $\hat{H}_{J, R} \equiv$ $-\sum_{j=1,2} \epsilon g_{j}(t) \hat{F}_{j}(t)$, where $\hat{F}_{j}(t) \equiv \lim _{\delta \rightarrow 0}\left[\hat{\mathcal{E}}\left(t, L_{j}-|\delta|\right)-\right.$ $\left.\hat{\mathcal{E}}\left(t, L_{j}+|\delta|\right)\right]$ is the force operator at the point $x=L_{j}[25]$. 
However, this method does not seem useful to obtain the reactive part of the motion force and the dynamical energy while the mirrors move. As before, in order to get those quantities we are led to use our Hamiltonian approach. This demands now considerable effort [22], e.g., generalizing the model [11], described by the Lagrangian density $\frac{1}{2}\left[\left(\partial_{t} \phi\right)^{2}-\left(\partial_{x} \phi\right)^{2}\right]-\sum_{j=1,2} \alpha_{j} \phi^{2} \delta\left[x-L_{j}(t ; \epsilon)\right]$, the Hamiltonian in the interaction picture has the form $\hat{H}_{I}(t)=$ $-\frac{\epsilon\left[g_{2}(t)-g_{1}(t)\right]}{L_{2}-L_{1}}\left[\int_{\mathbb{R}} d y\left(\partial_{y} \hat{\tilde{\phi}}_{I}(y)\right)^{2}+\sum_{j=1,2} \alpha_{j}\left(\hat{\tilde{\phi}}_{I}\left(L_{j}\right)\right)^{2}\right]+$ $\epsilon \sum_{j=1,2} \int_{\mathbb{R}} d y \frac{(-1)^{j} \dot{g}_{j}(t) \hat{\tilde{\xi}}_{I}(y)}{L_{2}-L_{1}}\left[\partial_{y} \hat{\tilde{\phi}}_{I}(y)\left(y-\bar{L}_{j}\right)+\frac{1}{2} \hat{\tilde{\phi}}_{I}(y)\right]+$ $\mathcal{O}\left(\epsilon^{2}\right)$ in terms of free quantum fields defined from an expansion in terms of left and right incident eigenfunctions. Our dissipative part of the motion force [22] coincides with the one obtained in [25]. For times $\tau$ larger than the stopping time, our quantum evolution operator is $\mathcal{T}^{\tau}=$ $\mathrm{Id}-i \int_{\mathbb{R}} d t \hat{H}_{I}(t)$. Using results from [11], we obtain explicitly that, for times $\tau$ larger than the stopping time, $\mathcal{T}^{\tau}=\mathrm{Id}+i \epsilon \sum_{j=1,2} \int_{\mathbb{R}} d t g_{j}(t) \hat{F}_{j}(t)$, as it should be. We see no basic obstruction to extend our procedure to higher dimensions and fields of any kind.

We should mention, to finish, that there are proposals to detect the radiated photons, although the reactive part and the possible deviations from conservative motion seem out of experimental reach yet [12].

We thank Gabriel Barton, Steve Fulling, and Roberto Onofrio for helpful discussions and correspondence. This work was supported in part by MEC (Spain), Projects No. MTM2005-07660-C02-01, No. BFM2003-00620, and No. PR2006-0145, and by AGAUR (Generalitat de Catalunya), Contract No. 2005SGR-00790.

*Electronic address: jaime.haro@upc.edu

†Presently at the Department of Physics and Astronomy, Dartmouth College, 6127 Wilder Laboratory, Hanover, NH 03755, USA.

Electronic addresses: elizalde@ieec.fcr.es; elizalde@ math.mit.edu

[1] V. V. Dodonov and A. B. Klimov, Phys. Rev. A 53, 2664 (1996).
[2] J. Y. Ji, H. H. Jung, J. W. Park, and K. S. Soh, Phys. Rev. A 56, 4440 (1997).

[3] M. Crocce, D. A. R. Dalvit, and F.D. Mazzitelli, Phys. Rev. A 66, 033811 (2002).

[4] R. Schützhold, G. Plunien, and G. Soff, Phys. Rev. A 65, 043820 (2002); G. Schaller, R. Schützhold, G. Plunien, and G. Soff, Phys. Rev. A 66, 023812 (2002).

[5] M. Ruser, J. Opt. B 7, S100 (2005).

[6] I. Brevik, K. A. Milton, S. D. Odintsov, and K. E. Osetrin, Phys. Rev. D 62, 064005 (2000).

[7] S. A. Fulling and P. C. W. Davies, Proc. R. Soc. A 348, 393 (1976).

[8] L.H. Ford and A. Vilenkin, Phys. Rev. D 25, 2569 (1982).

[9] G. Barton and C. Eberlein, Ann. Phys. (N.Y.) 227, 222 (1993); R. Gütig and C. Eberlein, J. Phys. A 31, 6819 (1998).

[10] C. Eberlein, J. Phys. I 3, 2151 (1993).

[11] G. Barton and A. Calogeracos, Ann. Phys. (N.Y.) 238, 227 (1995).

[12] W.-J. Kim, J.H. Brownell, and R. Onofrio, Phys. Rev. Lett. 96, 200402 (2006).

[13] M. M. Razavy and J. Terning, Phys. Rev. D 31, 307 (1985).

[14] C. K. Law, Phys. Rev. A 49, 433 (1994); 51, 2537 (1995).

[15] R. Schützhold, G. Plunien, and G. Soff, Phys. Rev. A 57, 2311 (1998).

[16] H. Johnston and S. Sarkar, J. Phys. A 29, 1741 (1996).

[17] M.-T. Jaekel and S. Reynaud, J. Phys. I 1, 1395 (1991).

[18] A. A. Grib, S. G. Mamayev, and V.M. Mostepanenko, Vacuum Quantum Effects in Strong Fields (Friedman Laboratory Publishing, Sankt Petersburg, 1994).

[19] G. T. Moore, J. Math. Phys. (N.Y.) 11, 2679 (1970).

[20] N. Obadia and R. Parentani, Phys. Rev. D 64, 044019 (2001).

[21] N.D. Birrell and C.P.W. Davies, Quantum Fields in Curved Space (Cambridge University Press, Cambridge, England, 1982).

[22] J. Haro and E. Elizalde (to be published).

[23] M.-T. Jaekel and S. Reynaud, Quantum Opt. 4, 39 (1992).

[24] D. A. R. Dalvit and P. A. Maia Neto, Phys. Rev. Lett. 84, 798 (2000); P. A. Maia Neto and D. A. R. Dalvit, Phys. Rev. A 62, 042103 (2000).

[25] M.-T. Jaekel and S. Reynaud, J. Phys. I 2, 149 (1992); 3, 1093 (1993); A. Lambrecht, M.-T. Jaekel, and S. Reynaud, Phys. Rev. Lett. 77, 615 (1996). 